\title{
PAPER
}

\section{The precedence effect for noise bursts of different bandwidths. II. Comparison of model algorithms}

\author{
Jonas Braasch and Jens Blauert \\ Institut für Kommunikationsakustik, Ruhr-Universität Bochum, \\ Universitätsstr. 150, 44780 Bochum, Germany \\ (Received 15 March 2003, Accepted for publication 27 May 2003 )
}

\begin{abstract}
In this investigation, different model algorithms were tested for their ability to simulate the precedence effect for ongoing (non-impulsive) noise-bursts of different bandwidths $(100 \mathrm{~Hz}$, $400 \mathrm{~Hz}$ and $800 \mathrm{~Hz}$ ). The psychoacoustical reference data-in which the perceived lateral position of a noise burst (200-ms duration, 20-ms $\cos ^{2}$-ramps, 500-Hz center frequency) in the presence of one reflection (inter-stimulus interval: $0.0 \mathrm{~ms}-0.4 \mathrm{~ms}$ ) was determined-were taken from a preceding paper on this investigation. It is shown that models which simulate the precedence effect by using the special characteristics of the auditory periphery or by focusing on the spectral dominance region fail when stimuli of longer duration than clicks are used, while a modified Lindemann model still shows satisfactory results. Furthermore, it was found that the trading ratio of ITDs and ILDs can be assumed to be constant for the stimuli tested. A discounting of ITD cues, as was found by Rakerd and Hartmann (1985), was not observed for the type of stimuli tested here. Instead, a discounting of the precedence effect occurred for some of the listeners when the bandwidth of the signals was very narrow. In the model simulation, it was not necessary to consider cross-frequency-band interaction like the second coincidence weighting of Stern et al. (1988), and it was sufficient to estimate the average of the outputs of the involved frequency bands.
\end{abstract}

Keywords: Binaural hearing, Precedence effect, Localization dominance, Model simulation

PACS number: 43.66.Mk, 43.66.Pn, 43.66.Ba, 43.55.Hy [DOI: 10.1250/ast.24.293]

\section{INTRODUCTION}

This paper is a companion to another paper by the same authors [1]. In that paper, which is in the following referred to as Paper I, results of a psychoacoustical experiment were presented. The aim of the experiment was to determine the ability of the auditory system to localize an ongoing signal ${ }^{1}$ - the so-called lead-in the presence of a single reflection-called the lag-in dependence of the bandwidth. As already indicated by earlier experiments [2], listeners' ability to form an auditory event in the direction from which the direct sound is presented improved with increasing bandwidth. This ability is usually referred to as "localization dominance" due to the precedence effect or (formerly) "law of the first wavefront."

In the following investigation, the performance of existing binaural models to predict the psychoacoustical results for ongoing stimuli in the presence of a single

\footnotetext{
${ }^{1}$ In this investigation we use the term "ongoing signal" in the sense of a "non-impulsive signal," but do not necessarily refer to a "never-ending signal."
}

reflection was tested. In some cases, the models had to be modified and extended to fit the need of this investigation. Although a number of models were developed to simulate the precedence effect, to the authors' knowledge, none of them were tested thoroughly on stimuli with a longer duration, but rather on experiments using click pairs or click trains. In the few cases, where models were applied to ongoing signals, only their principal functionality was demonstrated $[3,4]$. Several models that were developed to simulate certain aspects of the precedence effect can here be used for testing in the context of this paper.

Already in 1986, Lindemann proposed a model to simulate the effect of localization dominance. The model is based on a cross-correlation algorithm, but also includes elements of contralateral inhibition to suppress secondary peaks of the cross-correlation pattern [2,5]. Zurek [6], in a different approach, simply inhibited the influence of the lag by suppressing the information shortly after an onset has been detected. Wolf [3] used a different approach to simulate the localization dominance of the precedence effect; he determined the ITDs in the signal's envelope- 
onset slopes rather than the ITDs in the carriers of the stationary signal. The ITDs of the onset slopes are measured by comparing those points in time, at which the slopes in the left and right channels exceed a threshold. Recently, two further models have been introduced to explain psychoacoustical experiments using click pairs as stimuli. The first one by Tollin [7] achieves to simulate a number of experiments simply by focusing only on the spectral dominance region. The second model, proposed by Hartung and Trahiotis [8], demonstrates for click pairs that many experimental results can be explained as effects due to the auditory periphery. The novelty of the latter model is the integration of the Meddis hair-cell algorithm into a common cross-correlation model. In reference to the Lindemann model, the authors of both models emphasize that there is no need to integrate inhibitory elements to explain their data on click pairs. Also in the investigations of Braasch and Hartung [9,10], inhibitory elements were not necessary in order to simulate localization experiments in reverberant environments (mirror-image simulation of a small seminar room) with a cross-correlation model.

The model algorithms which are used in this study are described in the next section. In the simulations, primarily, the psychoacoustical results of Paper I were used as a reference (Section 3 and 4). In the test, the following models were compared: a simple cross-correlation algorithm, the cross-correlation model with an integrated Meddis hair-cell model as proposed by Hartung and Trahiotis, the cross-correlation model with spectral dominance weighting as developed by Tollin, and the crosscorrelation model with contralateral inhibition as proposed by Lindemann. Since ILD cues seem to play an important role in the psychoacoustical experiment in Paper I, algorithms to process ILD cues had to be included in the comparison. Here, a model based on excitation-inhibition cells was considered. The cross-correlation model with contralateral inhibition of Lindemann was modified by implementing a pre-compression stage in order to eliminate the influence of the ILDs, which were analyzed separately. Besides comparing existing algorithms and testing their suitability to process stimuli of longer duration in the presence of a reflection, the aim of this investigation was:

(i) To reveal, if the simulation of broadband stimuli requires interaction like the second coincidence weighting of Stern et al. [11], or if it is sufficient to estimate the average across the involved frequency bands

and

(ii) To investigate, if the influence of ITDs and ILDs on the position of the auditory event changes with decreasing bandwidth.

The latter aim was motivated by the outcome of an experiment by Rakerd and Hartmann [12]. In their experi- ment, listeners had to identify a sinusoidal sound source in the presence of a single reflection. The authors found that the ITD cues were discounted in certain cases. This finding led Rakerd and Hartmann to formulate their "plausibility hypothesis," which proposes that the auditory system determines sound-source positions from plausible cues only. Localization cues that are implausible are ignored to their hypothesis. In their experiment, ITD cues were frequently discounted by the auditory system for being implausible, because their values were unnaturally large or did not match the remaining cues (e.g., visual cues). Rakerd and Hartmann, therefore, assumed that the auditory system weights cues according to their plausibility.

\section{MODEL ALGORITHMS}

\subsection{Cross-correlation Algorithm}

In the first approach, a model based on a simple interaural cross-correlation algorithm and stages to simulate the auditory periphery were implemented, as shown in Fig. 1. The model is similar to the one proposed by Blauert and Cobben [13]. Basilar-membrane and hair-cell behavior are simulated with a gammatone-filter bank and a simple half-wave rectification. The gammatone-filter bank, which was described by Patterson et al. [14], consists, in this investigation, of 14 frequency bands from $244 \mathrm{~Hz}$ to $1,670 \mathrm{~Hz}$ center frequency in steps of one ERB. The sampling frequency $f_{\mathrm{s}}$ of the model was set at $48 \mathrm{kHz}$, and internal delays between $-1,000 \mu \mathrm{s}$ and $1,000 \mu \mathrm{s}$ were considered. After the half-wave rectification, the interaural cross correlation is estimated within each frequency band over the whole stimuli duration. In the decision device, the average interaural cross-correlation (ICC) functions were calculated after the peak of the ICC functions in each

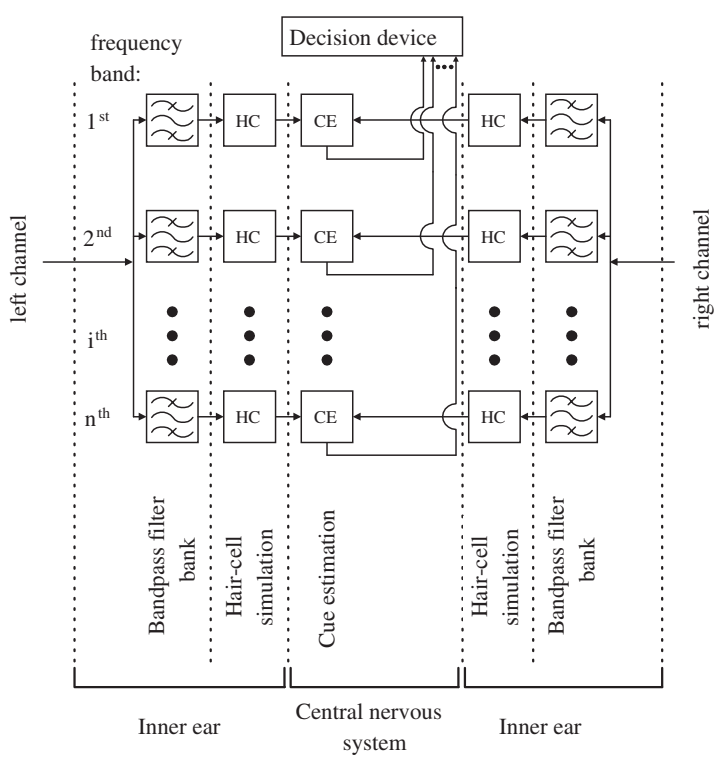

Fig. 1 General model structure of all evaluated localization algorithms. 
frequency band had been scaled to the average power of the stimulus in the left and right channel. The model either estimated the sound-source positions at the centroid of the averaged ICC function or at the position of the crosscorrelation peak. Even though this model was not specially designed to demonstrate the precedence effect, Blauert and Cobben [13] were able to show that it can account for some of its phenomena.

\subsection{ILD Algorithm Based on Excitation-Inhibition Cells}

An adequate method to process the ILDs in an analogous way to the processing of ITD cues with the cross-correlation algorithm is to use an array with excitation/inhibition (EI) cells. An algorithm with this characteristic was proposed by Breebaart et al. [15]. It employs an excitation-inhibition (EI) algorithm, based on the physiological findings of Reed and Blum [16]. In the investigation reported here, a version of Breebaart et al.'s algorithm was used, which was modified by Braasch [17] to analyze ILD cues only. ${ }^{2}$ In this algorithm, every cell has an excitatory and inhibitory input and is tuned to a certain ILD $\alpha$. The output of the EI cell $\mathbf{E}(\alpha)$ is estimated as follows:

$$
\mathbf{E}_{i}(\alpha)=\exp \left(\left[10^{\alpha / \mathrm{ILD}_{\max }} \sqrt{P_{i, 1}}-10^{-\alpha / \mathrm{ILD}_{\max }} \sqrt{P_{i, \mathrm{r}}}\right]^{2}\right),
$$

with $P_{i, 1}, P_{i, \mathrm{r}}$, being the power in the left and right channels and $i$ referring to the $i$ th frequency band.

In this model simulation, 61 EI-cells were used for each frequency band $i$. The ILD $\alpha$ was adjusted to values between -30 and $30 \mathrm{~dB}$ in steps of $1 \mathrm{~dB}$. The EI cells are implemented in each frequency band directly after the halfwave rectification. The peripheral stage is identical to the one of the cross-correlation model described in Section 2.1. Afterwards, the average of the EI-cell outputs of all frequency bands are calculated.

\subsection{Cross-correlation Algorithm with Integrated Meddis Hair-cell Model}

The only difference between the model of Hartung and Trahiotis [8] and the cross-correlation model that was described in Section 2.1 is the replacement of the halfwave rectification by the Meddis hair-cell model. For the latter, the setting to simulate a auditory nerve fiber with a highspontaneous rate [18] was chosen. Hence, the model was exactly like the model proposed by Hartung and Trahiotis [8] with two exceptions: (i) Hartung and Trahiotis originally used $100-\mathrm{kHz}$ sampling frequency and (ii) only internal delays from $-1,000 \mu \mathrm{s}$ to $1,000 \mu \mathrm{s}$ instead of $-1,500 \mu$ s to $1,500 \mu$ s were analyzed here. However, the

${ }^{2}$ In the original solution of Breebaart et al., the EI cells were tuned to both ILDs and ITDs, resulting in a 2-dimensional grid of cells for all combinations of different ITDs and ILDs. differences in sampling frequency and internal delays do not change the principal behavior. Our using of a sampling frequency of $48 \mathrm{kHz}$ only decreases the time resolution of the model, and the decreased range of the internal delay does not affect the estimated sound-source position for the tested type of stimuli since the latter is estimated in the model of Hartung and Trahiotis by determining the position of the cross-correlation peak rather than its centroid.

\subsection{Cross-correlation Algorithm with Spectral-Dom- inance Weighting}

Another approach to simulate the precedence effect was followed by Tollin [7]. Together with Henning, he found evidence that the positions of the listeners' auditory events strongly correlate with the ITD in the "dominance region" around $750 \mathrm{~Hz}$ [19]. The theory that the ITD cues in some frequency regions are more influential than the ITD cues in other regions has been proposed by Bilsen and Raatgever [20] many years before Tollin and Henning. Further, a frequency weighting to increase the influence of the ITDs in the dominance region-which was found to be between 300 and $750 \mathrm{~Hz}$ depending on the experimental task [11,20-22]—has been implemented in numerous models before. What is new about Tollin's and Henning's investigation is their assumption that for many listening tasks involving the precedence effect, the auditory system estimates the position of the auditory event exclusively from the information in the dominance region, provided that the signal is strong enough there. The information in the remaining frequency regions is then ignored in the model.

The computational model of Tollin [7] was implemented according to the description of the author. Basically, the structure was as shown in Fig. 1, but only one filter for each channel rather than a complete bandpass filter bank had to be included, because the model focuses exclusively on the dominance region. Since the filter width and shape has a considerable influence on the outcome of the model, we decided to use exactly the same gammatonefilter implementation that was originally used by Tollin to estimate the impulse response:

$$
h(f, t)=\left(\frac{f}{2 \pi}\right)^{n} \cdot t^{n-1} \cdot e^{-f t} \cdot \sin (2 \pi f t),
$$

with $f$ being the center frequency of the dominance region; $t$, the time; and $n=4$, the order of the filter. The signal was half-wave rectified after filtering, and afterwards the crosscorrelation for both channels was estimated. Originally, Tollin utilized a running cross-correlation algorithm with an exponential time-weighting function and estimated the position of the auditory events by averaging the outputs over time. By ending the evaluation by the cross-correlator 
directly after the signal offset, Tollin achieved to increase the localization dominance. The trick is to measure still some influence of the lead after the time window progressed to the lag, using the exponential window function, but turning off the cross-correlator immediately after the signal's ends to avoid that the declining tail of the exponential time window will pick up more information about the lag. Unfortunately, Tollin does not specify in his paper at which exact point in time the cross-correlator will be turned off. Is it directly after the signal ending or is the duration of the gammatone-filter impulse response still considered? If the latter is the case, it remains unclear how many coefficients of the infinite filter response have been calculated. In our work reported here, the average crosscorrelation function over the whole stimulus presentation is directly estimated, for two reasons:

(i) It does not seem plausible to us to switch off the cross-correlator directly after the signal. Why does the analysis not continue for a few milliseconds? At least, we observe a sluggishness of the auditory system in the order of several milliseconds for those types of tasks.

(ii) It does not make much difference whether the tail is included or not for ongoing signals.

Interaural level differences are considered in Tollin's model as well. They are computed directly from the average power in both channels and not as a running algorithm. The lateral position $\Omega$ is estimated using the equation:

$$
\Omega(f)=[\operatorname{ITD}(f)+\alpha \cdot \operatorname{ILD}(f)] / \tau_{0},
$$

with $\alpha=20 \mu \mathrm{s} / \mathrm{dB}$, the trading ratio of ITDs and ILDs and $\tau_{0}=60 \mu \mathrm{s}$, the normalization factor. In our implementation, the trading ratio $\alpha$ was set to $30 \mu \mathrm{s} / \mathrm{dB}$, and the normalization factor was adjusted to $1 \mu \mathrm{s}$.

\subsection{Cross-correlation Algorithm with Contralateral Inhibition and Pre-compression}

In the final approach, the model of Lindemann [5] was examined. In 1986, Lindemann was able to simulate the effect of localization dominance for bandpass-filtered click pairs [2], but he never investigated if his model would also process ongoing signals adequately. The novelty of Lindemann's algorithm is the introduction of contralateral-inhibition elements (static inhibition) into the crosscorrelation model. In the model, the signals in the delay lines for the left and right channel $l(m, n)$ and $r(m, n)$, that form the cross-correlation product $(k(m, n)=$ $l(m, n) \cdot r(m, n))$, are modified as follows:

$$
\begin{aligned}
& r(m+1, n-1)=r(m, n)\left[1-c_{\mathrm{s}} l(m, n)\right] \\
& l(m+1, n+1)=l(m, n)\left[1-c_{\mathrm{s}} r(m, n)\right]
\end{aligned}
$$

with $m$ being the index for discrete time. The variable $n$ is the index for internal delay and $c_{\mathrm{s}}$ refers to the static inhibition constant $\left(0 \leq c_{\mathrm{s}}<1\right)$.

Now, the signals of both channels inhibit each other, hence, reduce the amplitude of the signal in the opposite channel at the corresponding delay unit. In addition to the static inhibition, Lindemann also introduced a dynamic inhibition which he defined as follows:

$$
\begin{aligned}
\phi(m, n)= & c_{\mathrm{d}} \cdot k(m-1, n) \\
& +\phi(m-1, n) \cdot e^{-T_{\mathrm{v}} / T_{\text {inh }}}\left[1-c_{\mathrm{d}} \cdot k(m-1, n)\right] .
\end{aligned}
$$

with $\phi$ being the running, dynamic inhibition function. The variable $c_{\mathrm{d}}$ is the dynamic inhibition constant $\left(0 \leq c_{\mathrm{d}}<1\right)$, $T_{\mathrm{v}}$ is the time delay of a delay unit $\left(21 \mu \mathrm{s}=1 / f_{\mathrm{s}}\right)$, and $T_{\mathrm{inh}}$ represents the fade-off time constant of the nonlinear low pass.

Originally, Lindemann had only analyzed narrowband signals, and thus, no bandpass filter bank was required in his investigation. In our analysis, the gammatone-filter bank and halfwave rectification that were described in Section 2.1 are used in the model. The later extensions of the Lindemann model by Gaik [23] and Djelani [24] were not considered in this investigation. Gaik extended the Lindemann model to process natural combinations of ITDs and ILDs in head-related transfer functions (HRTFs). These are not needed in our investigation, because artificial stimuli presented through headphones have been used for the psychoacoustic data collection [1], and, therefore, an adequate processing for natural combinations of ITDs and ILDs is not necessary. Djelani applied a further, onsettriggered inhibition unit to the Lindemann model in order to simulate the build-up of the precedence effect for experimental data on click trains, which is not needed here, because the investigation does not focus on adaptational effects.

One characteristic of the Lindemann model is that the effective degree of inhibition depends on the signal's amplitude. To avoid the degree of inhibition being much lower in those frequency bands with less signal energy, the signal's maximum in each band was scaled to one. After the half-wave rectification, the cross-correlation patterns were computed and multiplied with the average power of the stimulus in the left and right channels measured in that frequency band. The cross-correlation patterns were added up, and the model estimated the positions of the sound sources regarding the centroid of the overall crosscorrelation pattern.

Another feature of the Lindemann model is the combined analysis of ITDs and ILDs. A side effect of the contralateral inhibition is a shift of the cross-correlation peak toward the channel with the higher energy. Since it is one of our aims to investigate whether the trading ratio of 


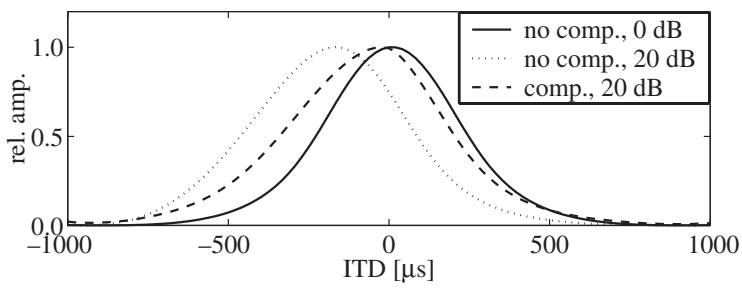

Fig. 2 Demonstration of the compression algorithm that was introduced in the Lindemann model to reduce the influence of the ILDs for a noise burst $(500-\mathrm{Hz}$ center frequency, $100-\mathrm{Hz}$ bandwidth, $0-\mu \mathrm{s}$ ITD). The solid line shows the average cross-correlation function, when the ILD of the signal is zero, and the dotted line, when the ILD of the signal is $20 \mathrm{~dB}$ (no compression). After inserting the compression stage, the peak moves-despite an ILD of $20 \mathrm{~dB}$ - almost to the center (dashed line).

ITDs and ILDs changes for the different conditions, it is better to analyze ITDs and ILDs in separate algorithms. We therefore decided to modify the Lindemann algorithm in such a way that it is almost independent of ILDs. Fortunately, the dependence on ILDs is quite low, and in fact Lindemann had to introduce monaural processors to enhance the influence of ILD cues. Besides omitting the monaural processors, the signal was compressed after the halfwave rectification by taking the signal to the power of 0.25 before it was scaled. In this way the influence of the ILDs is reduced furthermore, as can be seen in Fig. 2.

\subsection{ILD Algorithm with Temporal Inhibition}

To arrive at a model adequate to the Lindemann algorithm for the processing of ILD cues, the EI model, as was described in Section 2.2 was installed as a running algorithm with inhibition units. Therefore, Eq. (1) had to be modified to:

$$
\begin{aligned}
\mathbf{E}_{i}(m, \alpha)= & \exp \left(\left[10^{\alpha / \mathrm{ILD}_{\max }} \sqrt{P(m)_{i, 1}}\right.\right. \\
& \left.\left.-10^{-\alpha / \mathrm{ILD}_{\max }} \sqrt{P(m)_{i, \mathrm{r}}}\right]^{2}\right),
\end{aligned}
$$

with $P(m)_{i, 1}, P(m)_{i, \mathrm{r}}$, the power in the left and right channels, and $i$ and $m$ referring to the $i$ th frequency band and the $m$ th time slot. Before the outputs of the halfwave rectification were sent to the inputs of the EI algorithm, they were convolved with a Hamming window of 10-ms duration to acknowledge the effect of binaural sluggishness. Afterwards, the outputs of the EI algorithm were down-sampled to a resolution of $1 \mathrm{~ms}$.

The inhibition function $\mathbf{E}_{\text {inh }}(m)$ and the new, inhibited function $\mathbf{E}_{\text {new }}(m)$ were calculated iteratively as:

$$
\begin{aligned}
\mathbf{E}_{\text {inh }}(m, \alpha)= & {\left[\max \left(\mathbf{E}_{\text {new }}(m-1, \alpha)\right)\right.} \\
& \left.-\mathbf{E}_{\text {new }}(m-1, \alpha)\right] \cdot c_{1} \\
& +\mathbf{E}_{\text {inh }}(m-1, \alpha) \cdot c_{2} \\
\mathbf{E}_{\text {new }}(m, \alpha)= & \mathbf{E}(m, \alpha)-\mathbf{E}_{\text {inh }}(m, \alpha)
\end{aligned}
$$

with $\quad c_{1}$ and $c_{2}$ being two inhibition constants $\left(0 \leq c_{1}, c_{2}<1\right)$. After having calculated each step, negative values of $\mathbf{E}(m)_{\text {inh }}$ and $\mathbf{E}(m)_{\text {new }}$ were set to zero, as a negative activity of the cells would be invalid for a physiologically oriented model.

\subsection{Decision Device}

If not indicated otherwise, the estimated sound-source position is determined by calculating the centroid of the average cross-correlation function or the centroid of the average activities of the excitation/inhibition cells. Only in one specified case, the sound-source position is determined by the position of the cross-correlation peak's maximum. The model units [MU] for the lateralization are chosen so that the perceived lateral position for the $-300-\mu \mathrm{s}$ reference stimulus is -1 , the lateral position for the $0-\mu \mathrm{s}$ reference stimulus is zero, and the perceived lateral position for the $300-\mu$ s reference stimulus is 1 . In order to estimate the lateralization $\Omega$ for the ILD analysis, the trading ratio between ITDs and ILDs was determined by the ILD of the acoustic pointer that was adjusted by the listeners to match the reference stimuli [1]. In this context, $300 \mu$ s corresponds to approximately $10 \mathrm{~dB}$. An ILD of $\pm 10 \mathrm{~dB}$ is equivalent to a model unit of \pm 1 and an ILD of $0 \mathrm{~dB}$ corresponds to a model unit of 0 .

The lateralization $\Omega$ of the lead-lag pairs was also estimated on the basis of a combined ITD-ILD analysis. For this purpose, the lateralization $\Omega$ was the sum of the results for one of the cross-correlation models and the correspondent EI model:

$$
\Omega[\mathrm{MU}]=\frac{\mathrm{ITD}}{\mathrm{ITD}_{300 \mu \mathrm{s}}}+\frac{\mathrm{ILD}}{10 \mathrm{~dB}} .
$$

Since double peaks occur in the cross-correlation functions, the ITD for the $300 \mu \mathrm{s}$ reference stimulus $\left(\mathrm{ITD}_{300 \mu s}\right)$ is not $300 \mu \mathrm{s}$ when determined through the centroid. For the excitation/inhibtion cell, on the other hand, it does not make a difference if the ILD is determined by the centroid or the position of the peak's maximum. Double peaks are not observed in this analysis, if the observed range of ILDs is large enough.

\section{STIMULI}

Before reporting on the evaluation of the model algorithms that were introduced in the previous section, the test material to evaluate the model will now be described briefly. The same test signals were used to test the model algorithms that have been used in the psychoacoustic experiments in Paper I. The lead and lag were bandpass-filtered, frozen white-noise bursts (200-ms duration, 20-ms $\cos ^{2}$-ramps). Since all tested models are perfectly symmetrical in their architecture regarding the left and the right channels, the ITD of the lead was always 
adjusted to $300 \mu \mathrm{s}$ and the lag to $-300 \mu \mathrm{s}$, while the conditions were omitted, where the ITD of the lead was adjusted to $-300 \mu$ s and the lag to $300 \mu \mathrm{s}$.

The bandpass filtered noise was generated digitally at a sampling frequency of $48 \mathrm{kHz}$ and 16-bit resolution. The center frequency of the bandpass filter was kept constant at $500 \mathrm{~Hz}$, and the following bandwidths were tested: $100 \mathrm{~Hz}$, $400 \mathrm{~Hz}$, and $800 \mathrm{~Hz}$. For the inter-stimulus interval (ISI), the delay between the onsets of lead and lag, the following values were considered: $0.0 \mathrm{~ms}$ and $1.0 \mathrm{~ms}$ to $4.0 \mathrm{~ms}$ in steps of $0.5 \mathrm{~ms}$. In the reference condition, only the lead with an ITD of $-300 \mu \mathrm{s}, 0 \mu \mathrm{s}$, or $300 \mu \mathrm{s}$ was analyzed by the model.

\section{MODEL-SIMULATION RESULTS}

\subsection{Cross-correlation Algorithm}

The left panels of Fig. 3 show the results of the simulation using the cross-correlation model. The results for the stimuli with a bandwidth of $100 \mathrm{~Hz}$ are shown in the top-left panel. In the center-left panel the simulation for the stimulus with $400-\mathrm{Hz}$ bandwidth is depicted, and in the bottom-left panel the simulation for the stimulus with 800 $\mathrm{Hz}$ bandwidth is given. The ISI is plotted along the $x$-axis, the computed lateralization is displayed along the $y$-axis. The graphs clearly show that the output of a simple crosscorrelation model does not represent the psychoacoustical data shown in the very right panels. Unfortunately—but not unexpectedly- the simulated data show no variation at all and always fall at zero, the average between lead and lag.

\subsection{ILD Algorithm Based on Excitation-Inhibition Cells}

The results of the model are shown in the second column from the left in Fig. 3, analogously to the results of the cross-correlation model. For the stimuli with a bandwidth of $100 \mathrm{~Hz}$, the simulation results of the EI model are very similar to the psychoacoustical results for the Type-II response pattern ('+'s in the top-right panel). This is in agreement with our statement in Paper I that in those cases, the listeners simply based their localization judgements on the ILDs of the ongoing part of the stimuli. In the top panel second from right of Fig. 3, the combined results of the EI model and the cross-correlation model, that was discussed in Section 2.1, are shown. Both cues, ILDs and ITDs are weighted equally in this combined analysis. The output of this combined model could be considered surprising as it would be expected that the magnitude of the results would be approximately half the value of the EI model, since the results of the crosscorrelation model are always near zero. It must be considered, however, that the acoustic pointer, the adjustment of which was used to determine the trading ratio of ITDs and ILDs, had a fixed ITD of $0 \mu \mathrm{s}$. The results of the combined model fit the results of the Type-II response

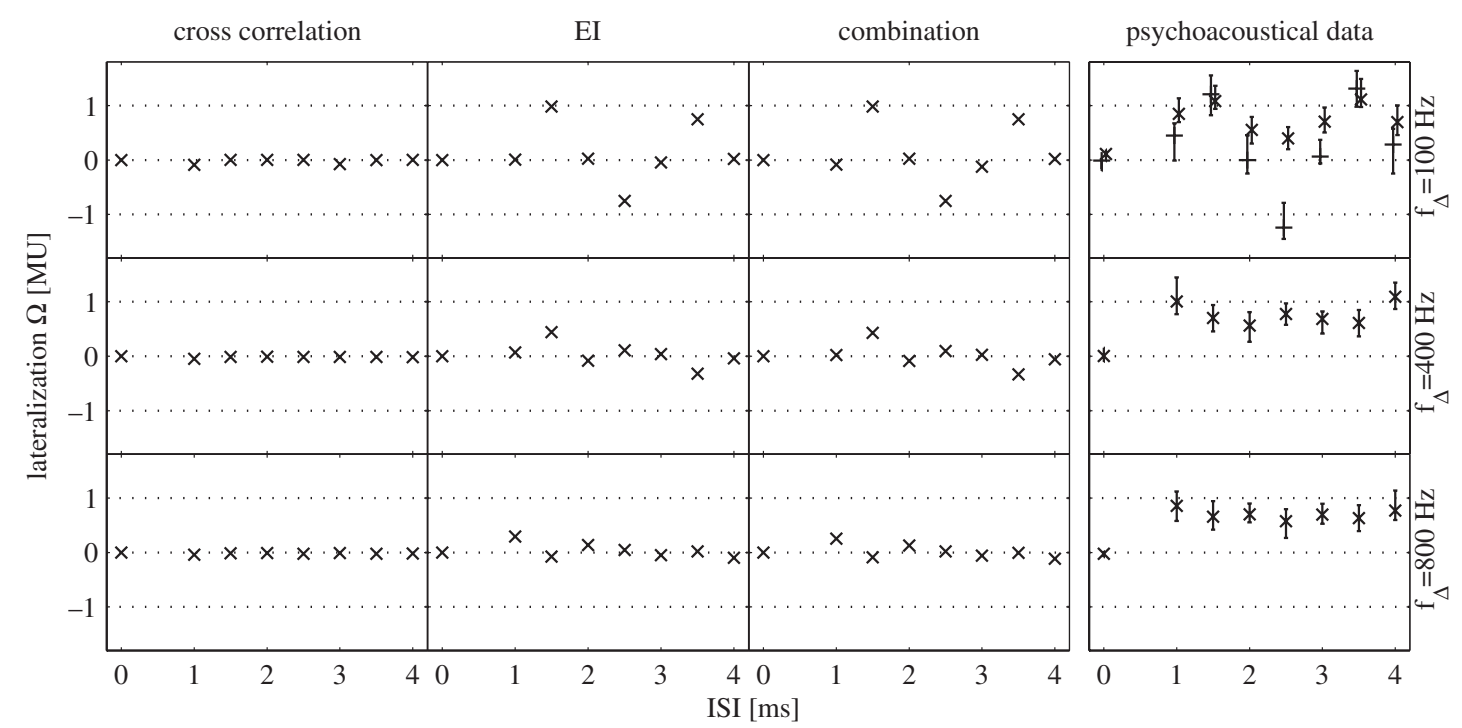

Fig. 3 Model performance of the cross-correlation and EI model without inhibition stage for the lateralization of a noise burst in the presence of a reflections for different ISIs and bandwidths (from top to bottom row): $100 \mathrm{~Hz}, 400 \mathrm{~Hz}, 800 \mathrm{~Hz}$. In the left panels, the results for the cross-correlation model are shown. The results for the EI model are depicted in the second column from the left. The combination of both, is given in the second column from the right. The very right panels depict the medians of the results obtained for six listeners as measured in Paper I. The data have been pooled over the conditions in which the ITD of the lead was set at $300 \mu$ s and the conditions, in which the ITD of the lead was set at $-300 \mu \mathrm{s}$. The results of the latter were mirrored at the origin of the $x$-axis. The top panel displays results for the $100-\mathrm{Hz}$ signal-bandwidth condition (Type I, ' $x$ 's; Type II, '十's), the center panel showed the results for the 400-Hz signalbandwidth condition (all Type I), and the bottom panel depicts the results for the 800-Hz signal-bandwidth condition (all Type I). 
pattern, therefore, supporting the hypothesis which was raised in Paper I, namely, that in those cases the precedence effect has a less important role.

For the remaining experimental conditions, $400-\mathrm{Hz}$ signal bandwidth and $800-\mathrm{Hz}$ signal bandwidth, the results of the combined model do not match any of the psychoacoustical results. The magnitude in the variance of the auditory events with the ISI may decline with increasing bandwidth, but the responses are always centered near zero and not near the lead.

\subsection{Cross-Correlation Algorithm with Integrated Meddis Hair-cell Model}

In the two models considered so far, only the responses for the stimuli with $100-\mathrm{Hz}$ bandwidth could be partly simulated, namely those of Type II. In the current section, it is investigated if those data, in which localization dominance was observed (Type-I data), can be simulated by a cross-correlation model after including the hair-cell model by Meddis $[18,25,26]$, as was proposed by Hartung and Trahiotis [8].

The results of the analysis are shown in the left graph of Fig. 4 with values near zero, the results are very similar to those of the previous implementation of the cross-correlation model (left panels of Fig. 3) for most conditions. A few exceptions exist, namely values with a large magnitude $( \pm 3.3 \mathrm{MU})$ at an ISI of $1 \mathrm{~ms}$ and at $100 \mathrm{~Hz}$ bandwidth also at an ISI $3.0 \mathrm{~ms}$. These outliers are not triggered by the Meddis model, but are rather a side effect of the decision device. Recall that in the model approach of Hartung and Trahiotis [8] the position of the sound source is determined
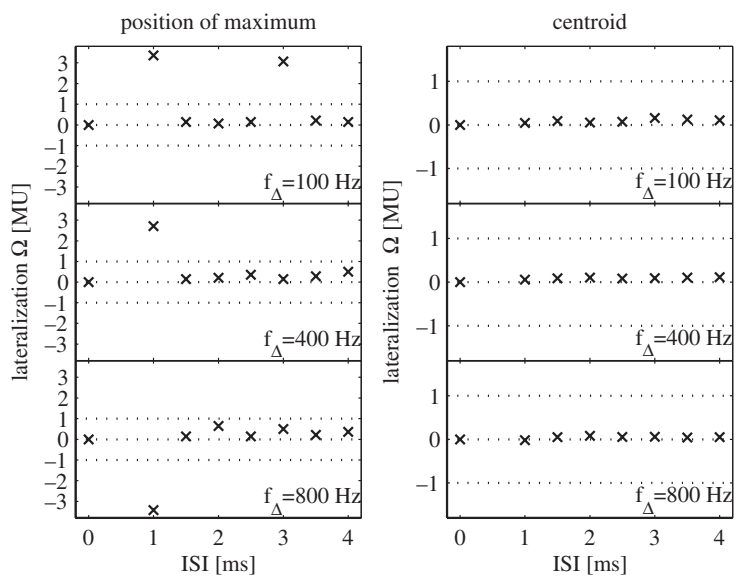

Fig. 4 Model performance of the cross-correlation including the Meddis hair-cell model for the lateralization of a noise burst in the presence of a reflections for different ISIs and bandwidths (from top to bottom row): $100 \mathrm{~Hz}, 400 \mathrm{~Hz}, 800 \mathrm{~Hz}$. In the left graph, the estimated position was determined by the position of the cross-correlation peak. In the right graph, the results for the centroid are depicted. by the position of the highest cross-correlation peak, rather than its centroid (Fig. 4, right panel). At an ISI of $1.0 \mathrm{~ms}$, the interfering lead and lag are out of phase regarding the left and right channels, and for this condition two crosscorrelation peaks appear at $-1,000 \mu \mathrm{s}$ and $1,000 \mu \mathrm{s}$, which is equivalent to $\mp 3.33 \mathrm{MU}$. The decision device makes its decision on the basis of the minimally higher of the two peaks. The model results become very similar to those of the previous implementation of the cross-correlation model (left panels of Fig. 3) for all conditions, when the soundsource positions are estimated by the centroid (Fig. 4, right panel). Unfortunately, the model is not able to simulate the localization dominance anymore, when the sounds have a considerably longer duration than clicks.

\subsection{Cross-correlation Algorithm with Spectral-Dom- inance Weighting}

The center frequency of the filter was chosen to be $750 \mathrm{~Hz}$, corresponding to the center frequency of the spectral dominance region. In the $100-\mathrm{Hz}$ signal bandwidth condition, the center frequency of the filter was adjusted to $500 \mathrm{~Hz}$, as in this condition the signal has no energy at $750 \mathrm{~Hz}$.

Even though the model of Tollin is able to predict the effect of localization dominance for a number of experiments using clicks as stimuli, it is not able to explain the data when ongoing signals are used (Fig. 5). The simulation results for the $100-\mathrm{Hz}$ bandwidth condition (top panels) are almost exactly like the results for the simple cross-correlation and EI model. This is not surprising, since the bandwidth of the stimuli is only $100 \mathrm{~Hz}$ wide, so that both models do basically the same analysis.

For the other two conditions (center panels, $400-\mathrm{Hz}$ signal bandwidth; bottom panels, $800-\mathrm{Hz}$ signal bandwidth), the results of Tollin's are still very similar to the $100-\mathrm{Hz}$ signal-bandwidth condition, only that here the output of the EI model does not vary with the inverse of the signal's center frequency, but rather with the inverse of the center frequency of the applied gammatone filter.

\subsection{Cross-correlation Algorithm with Contralateral Inhibition and Pre-compression}

In the model simulation, several combinations of the values for the static and the dynamic inhibition constants $c_{\mathrm{s}}, c_{\mathrm{d}}$ and $T_{\text {inh }}$ were tested. The best results were obtained, when $c_{\mathrm{s}}$ was set to $0.9, c_{\mathrm{d}}$ to 0.5 , and $T_{\mathrm{inh}}$ to $50 \mathrm{~ms}$. The model results for this setting are shown in the left panels of Fig. 6 from top to bottom: $100 \mathrm{~Hz}, 400 \mathrm{~Hz}$ and $800 \mathrm{~Hz}$. In all three conditions, the output of the modified Lindemann algorithm is fairly independent from the ISI. The only exceptions are the conditions for an ISI of $0.0 \mathrm{~ms}$, but in these conditions, the results have to be zero for symmetrical reasons because there is no time delay between 


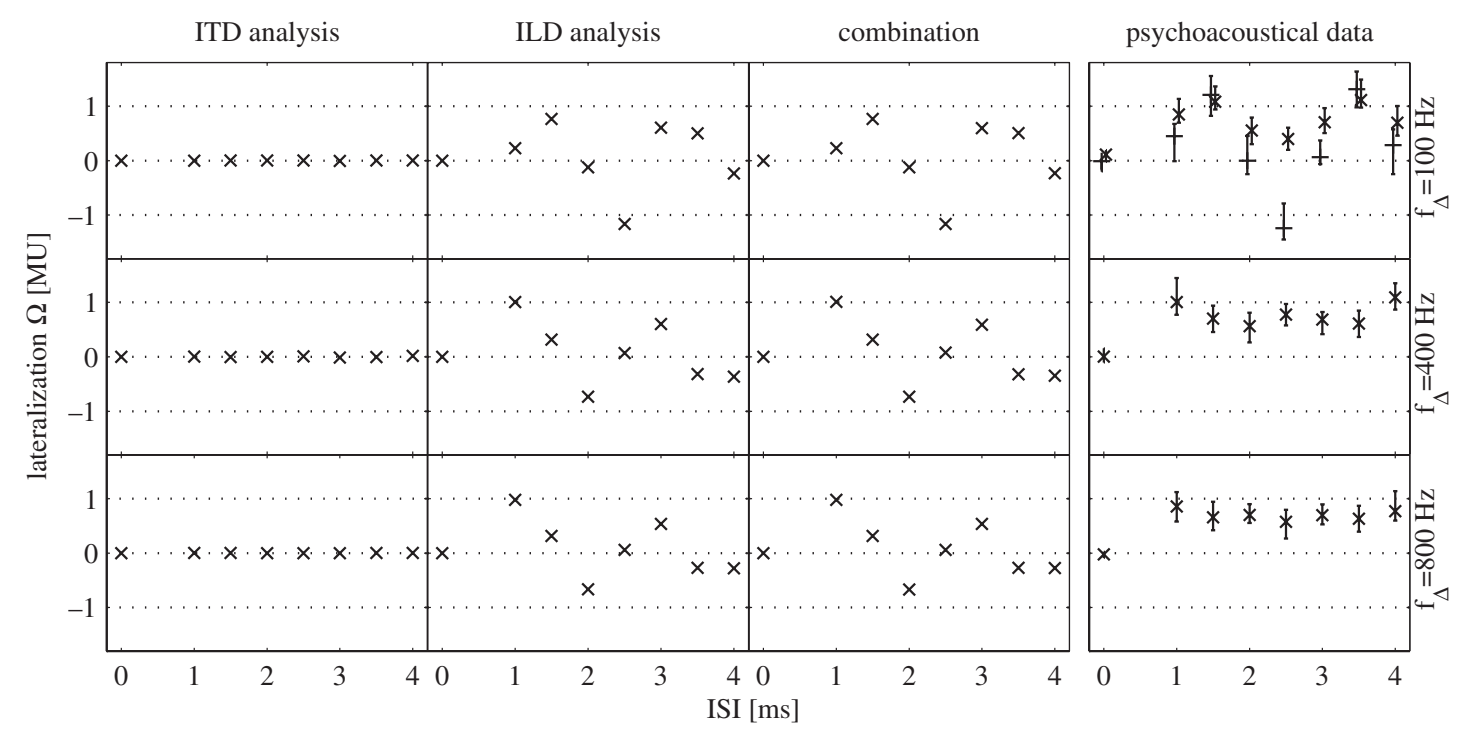

Fig. 5 Model performance of Tollin's model without inhibition stage for the lateralization of a noise burst in the presence of a reflection for different ISIs and bandwidths (from top to bottom row): $100 \mathrm{~Hz}, 400 \mathrm{~Hz}, 800 \mathrm{~Hz}$. In the left panels, the results for the ITD analysis are shown. The results for the ILD analysis are depicted in the second column from the left. The combination of both, is given in the second column from the right. The very right panels depict the medians of the psychoacoustic results as in Fig. 3.

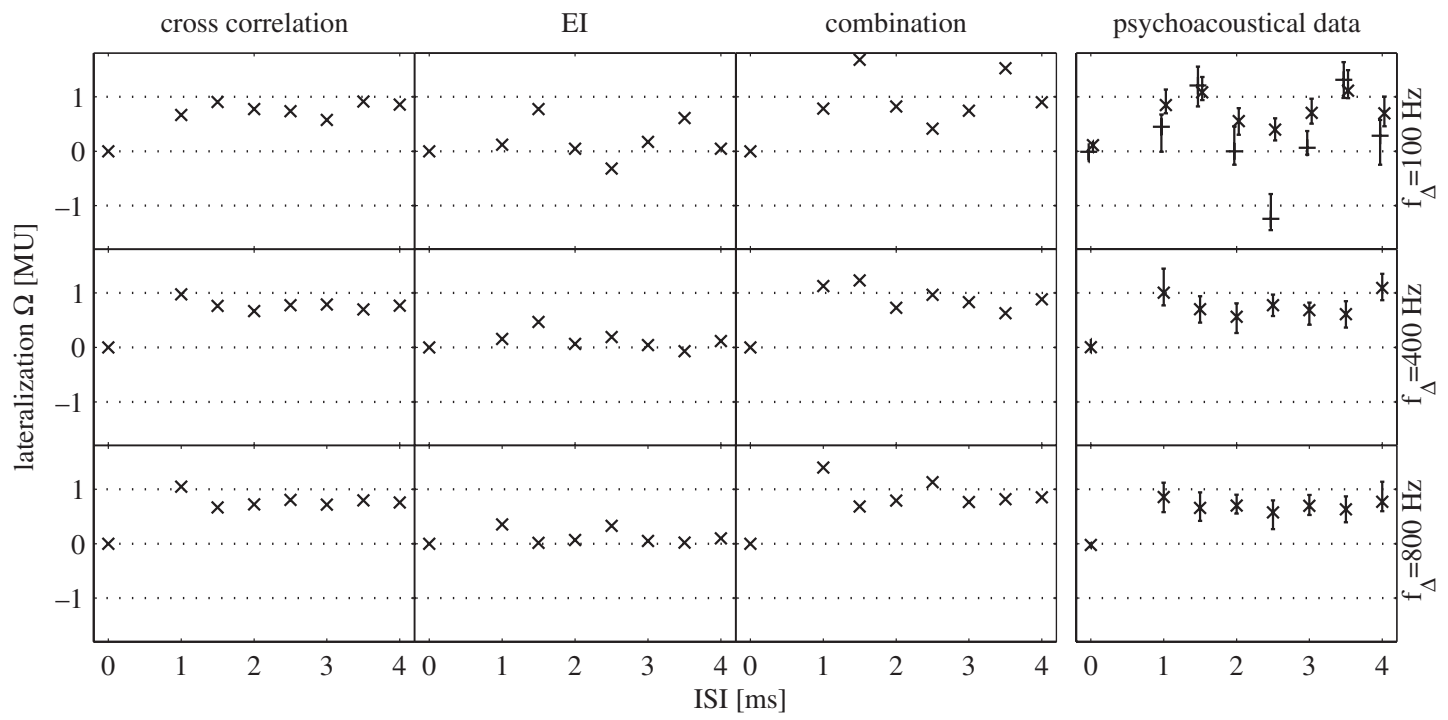

Fig. 6 Same as Fig. 3, but inhibition stages were implemented into both the cross-correlation and the EI algorithms (modified Lindemann model).

lead and lag. Effectively, the contralateral inhibition causes an onset enhancement by keeping the peak of the crosscorrelation pattern on track of the first activated peak (Fig. 7). In contrast to a simple onset enhancement algorithm, the Lindemann algorithm can only keep the peak on track if there is energy left at this internal delay. If not, the position of the peak will move considerably or the peak will vanish after the energy of the signal is reduced. In contrast, a simple onset enhancement model, like the one of Wolf [3], for example, ignores what happens after an onset is found, until the detection of a new onset takes place.

After being partly successful with the modified Linde- mann algorithm - the effect of localization dominance appeared, but the estimated sound-source position did not vary with the ISI in the $100-\mathrm{Hz}$ signal-bandwidth condition-it was investigated if an inhibition mechanism can be successfully integrated into the EI model that was described in Section 2.2.

\subsection{ILD Algorithm with Temporal Inhibition}

The model results for this setting are shown in the panels second from the left in Fig. 6. In the model simulations, the inhibition constants $c_{1}$ and $c_{2}$ were set to 0.9 and 0.5 . In the top panel, the model results for the 100- 

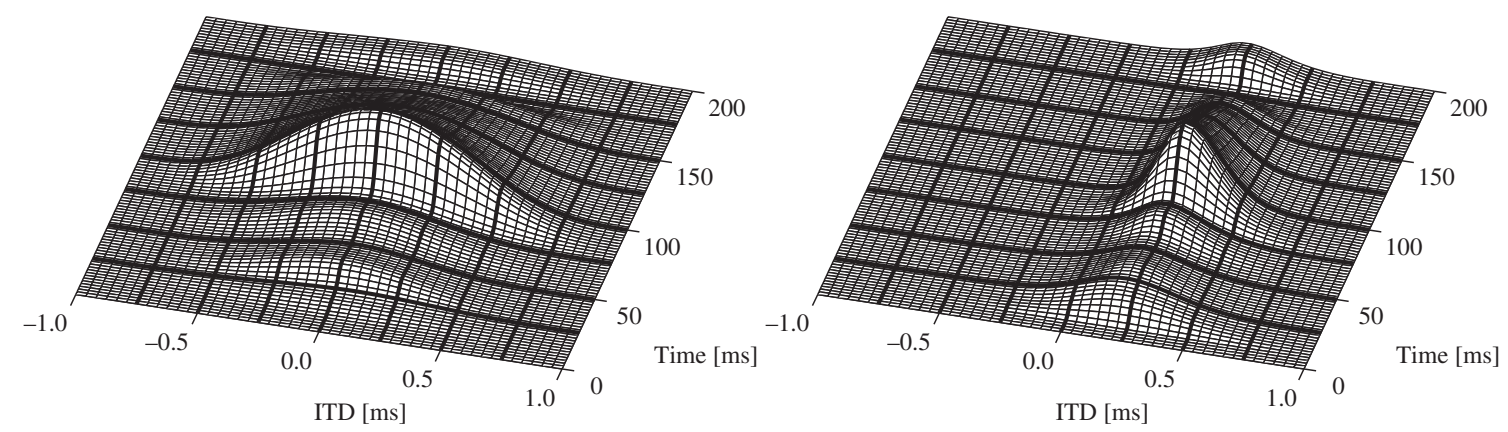

Fig. 7 Output of the Lindemann algorithm (right panel) in comparison to the plain ICC algorithm without inhibition (left panel) for a narrowband noise $(500-\mathrm{Hz}$ center frequency, $100-\mathrm{Hz}$ bandwidth, 300- $\mu$ s ITD) in the presence of a reflection (-300- $\mu$ s ITD, 1.5-ms inter-stimulus interval).

$\mathrm{Hz}$ signal-bandwidth condition are depicted. The variation of the estimated sound-source positions with the ISI is significantly reduced compared to the EI algorithm without inhibition. Also in this algorithm, the inhibition causes an onset enhancement, and the effect of localization dominance appears. Recall that both the lead and the lag have their ILD adjusted to zero, and only through the interference of both, ILDs occur. If the output were fully independent of the lag (localization dominance), it should be always at zero.

The results of the combined model (combination of the modified Lindemann model and the EI model with inhibition) for the 100-Hz signal-bandwidth condition are shown in the top panel, second from the right. Except for the values of the estimated position of the sound-source position being relatively high for ISIs of $1.5 \mathrm{~ms}$ and $3.5 \mathrm{~ms}$, the model result match the Type-I results of the psychoacoustical experiment quite well. The simulation suggests that in this condition, the ITD cues shift the auditory events toward the lead (localization dominance), while the ILD cues cause the ISI-dependent variation.

These variations decrease, when the bandwidth of the stimuli is increased from $400 \mathrm{~Hz}$ to $800 \mathrm{~Hz}$ (Fig. 6). As already discussed in Paper I, this result is found because the ILDs that occur in the frequency bands average-out each other. The results for the combined model are shown in the center panel, second from the right $(400-\mathrm{Hz}$ signalbandwidth condition) and the bottom panel, second from the right $(800-\mathrm{Hz}$ signal-bandwidth condition). In both conditions, the estimated sound-source position is fairly constant for ISIs between $1.0 \mathrm{~ms}$ and $4.0 \mathrm{~ms}$, as it was already found in the psychoacoustical experiments. Furthermore, even small features like the slightly higher value at an ISI of $1.0 \mathrm{~ms}$ in the $800-\mathrm{Hz}$ signal-bandwidth condition, are found in the simulation results.

Figure 8 shows the correlograms for the condition of the psychoacoustic experiment in which the ISI of the lead is set at $3.5 \mathrm{~ms}$ and the bandwidth is adjusted to $800 \mathrm{~Hz}$, when utilizing different cross-correlation algorithms. After integrating the Meddis hair-cell model (top-right panel), for example, the width and positions of the cross-correlation peaks throughout frequency are changed much less (compared to the plain cross-correlation model, top-left panel) than it is the case when introducing a contralateral inhibition mechanism (bottom-left panel). For data on click pairs, much of the change in the data after employing the Meddis hair-cell algorithm into the cross-correlation model, can be explained by a modified weighting of the outputs of the different frequency bands. In the present test situation, this effect does not change the average crosscorrelation pattern much. In case of the cross-correlation model with contralateral inhibition, a different weighting of the information across frequency is not important, but rather the cross-correlation peaks in each frequency band appear at the side of the lead.

\section{DISCUSSION AND CONCLUSIONS}

Even though all evaluated cross-correlation algorithms were able to simulate experiments with click pairs, only the cross-correlation model with contralateral inhibition is able to account for localization dominance when stimuli of reasonable duration are used. For noise bursts of longer duration, an integration of the Meddis hair-cell model is not sufficient to demonstrate the precedence effect. Nevertheless, this model gives very good results for click pairs and its architecture is plausible, one might think to include elements of contralateral inhibition into this approach. In a new model approach, one could imagine to use peripheral effects to explain the localization dominance for the onsets of the signal and to use inhibitory elements to account for the localization dominance in the ongoing part. This model approach could be possibly used to explain the build-up of the precedence effect, accounting that the inhibition increases with the exposure time of the stimulus.

The explanation of the localization-dominance effect by spectral dominance weighting, on the contrary, were not very promising. Not did it fail completely to show the influence of increasing bandwidth (Section 4.4), but also 

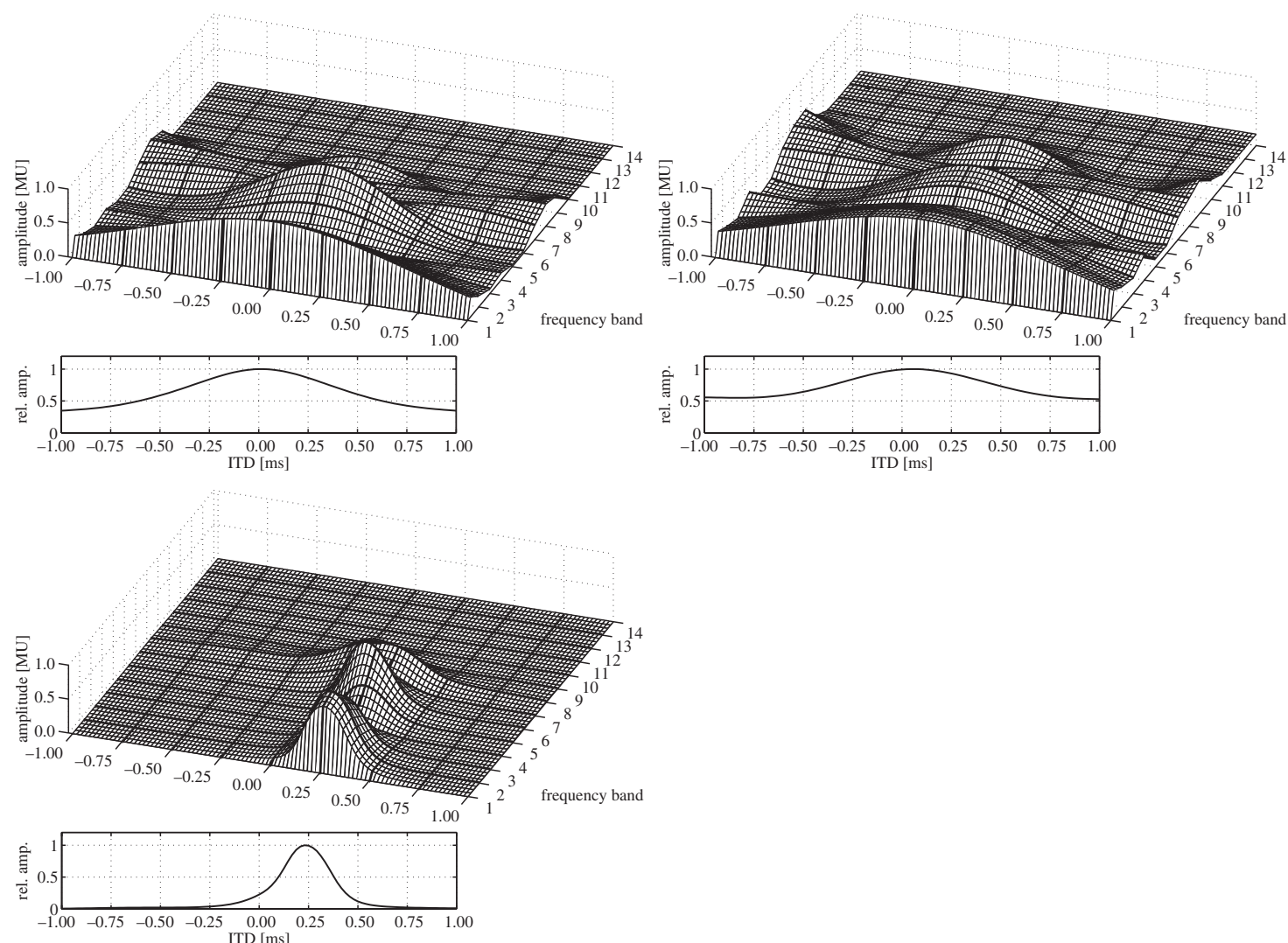

Fig. 8 Correlograms of the model results for the test condition with 3.5-ms ISI and $800-\mathrm{Hz}$ bandwidth. The results for the following model algorithms are shown: plain cross-correlation model (top-left panel), cross-correlation model with Meddis haircell model (top-right panel), cross-correlation model with contralateral inhibition and pre-compression stage (bottom-left panel). Below each correlogram, the average of all frequency bands is plotted.

the change of the center frequency by only a few Hertz changes the model results dramatically. In addition, it must be doubted that the center frequency of the spectral dominance region is constant within a few Hertz for different listeners.

The results of the simulation also revealed that both the analysis of ITDs and ILDs are necessary to simulate the psychoacoustic results. In principle, ITD cues can be accounted for the perceived sideness of the stimulus at the side of the lead, while ILD cues cause the variation of the auditory event with the ISI.

In the model simulation using the modified Lindemann model, the trading ratio of ITDs and ILDs was kept constant for the different tested bandwidths. It can, therefore, be assumed that a discounting of ITD cues, as observed by Rakerd and Hartmann [12] for a sinusoidal tone in the presence of a reflection, was not observed with our stimuli. Instead, it was shown in the model simulations that by reducing the inhibition factor to zero a discounting of the precedence effect was observed for some of the listeners (Type-II response patterns, $100-\mathrm{Hz}$ bandwidth condition). It should also be noted that it was sufficient to use a purely signal-driven model (bottom-up) to simulate the psychoacoustical experiments of Paper I and that it was not necessary to implement stages to simulate cognitive processes.

It is of note that it was sufficient to estimate the average across the outputs of the involved frequency bands. For the employed stimuli, it was not necessary to take crossfrequency-band interaction like the second coincidence weighting of Stern et al. [11] into account. It may well be that large parts of the precedence effect will finally be found to be less complicated to explain than often thought.

\section{ACKNOWLEDGEMENT}

We would like to thank the anonymous reviewers of this paper for their helpful comments and suggestions during the revision process and John Worley for proofreading the manuscript. This work was financed by the Deutsche Forschungsgemeinschaft (Grant No. Bl 189/23$3)$.

\section{REFERENCES}

[1] J. Braasch, J. Blauert and T. Djelani, "The precedence effect for noise bursts of different bandwidths. I. Psychoacoustical data," Acoust. Sci. \& Tech., 24, 233-241 (2003).

[2] W. Lindemann, "Extension of a binaural cross-correlation model by contralateral inhibition. II. The law of the first wave front," J. Acoust. Soc. Am., 80, 1623-1630 (1986). 
[3] S. Wolf, Untersuchungen zur Lokalisation von Schallquellen in geschlossenen Räumen, Dissertation, Ruhr-Universität Bochum (1991).

[4] J. Huang, N. Ohnishi, X. Guo and N. Sugie, "Echo avoidance in a computational model of the precedence effect," Speech Commun., 27, 223-233 (1999).

[5] W. Lindemann, "Extension of a binaural cross-correlation model by contralateral inhibition. I. Simulation of lateralization of stationary signals," J. Acoust. Soc. Am., 80, 1608-1622 (1986).

[6] P. M. Zurek, "The precedence effect," in Directional Hearing, W. A. Yost and G. Gourevitch, Eds. (Springer-Verlag, New York, 1987), pp. 85-105.

[7] D. J. Tollin, "Computational model of the lateralisation of clicks and their echoes," in Proc. the NATO Advanced Study Institute on Computational Hearing (Il Ciocco), S. Greenberg and M. Slaney, Eds. (1998), pp. 77-82.

[8] K. Hartung and C. Trahiotis, "Peripheral auditory processing and investigations of the "precedence effect" which utilize successive transient stimuli," J. Acoust. Soc. Am., 110, 15051513 (2001).

[9] J. Braasch and K. Hartung, "Localization in the presence of a distracter and reverberation in the frontal horizontal plane. I. Psychoacoustical Data," Acustica/Acta Acustica, 88, 942-955 (2002).

[10] J. Braasch, "Localization in the presence of a distracter and reverberation in the frontal horizontal plane. II. Model algorithms," Acustica/Acta Acustica, 88, 956-969 (2002).

[11] R. M. Stern, A. S. Zeiberg and C. Trahiotis, "Lateralization of complex binaural stimuli: A weighted image model," $J$. Acoust. Soc. Am., 84, 156-165 (erratum: J. Acoust. Soc. Am., 90, 2202) (1988).

[12] B. Rakerd and W. M. Hartmann, "Localization of sound in rooms. II. The effect of a single reflecting surface," J. Acoust. Soc. Am., 78, 524-533 (1985).

[13] J. Blauert and W. Cobben, "Some consideration of binaural cross correlation analysis," Acustica, 39, 96-104 (1978).

[14] R. D. Patterson, M. H. Allerhand and C. Giguère, "Timedomain modeling of periphal auditory processing: A modular architecture and software platform," J. Acoust. Soc. Am., 98, 1890-1894 (1995).

[15] J. Breebaart, S. van de Par and A. Kohlrausch, "Binaural processing model based on contralateral inhibition. I. Model setup,” J. Acoust. Soc. Am., 110, 1074-1088 (2001).

[16] M. C. Reed and J. J. Blum, "A model for the computation and encoding of azimuthal information by the lateral superior olive," J. Acoust. Soc. Am., 88, 1442-1453 (1990).

[17] J. Braasch, "Localization in the presence of a distracter and reverberation in the frontal horizontal plane: III. The role of interaural level differences," Acustica/Acta Acustica, 89, 674692 (2003).

[18] R. Meddis, M. J. Hewitt and T. M. Shakleton, "Implementation details of a computational model of the inner hair-cell auditory-nerve synapse," J. Acoust. Soc. Am., 87, 1813-1816 (1990).
[19] D. J. Tollin and G. B. Henning, "Some aspects of the lateralisation of echoed sound in man. II. The role of the stimulus spectrum," J. Acoust. Soc. Am., 105, 838-849 (1999).

[20] F. A. Bilsen and J. Raatgever, "Spectral dominance in binaural lateralization," Acustica, 28, 131-132 (1973).

[21] J. Raatgever, On the Binaural Processing of Stimuli with Different Interaural Phase Relations, Dissertation, Technische Hogeschool, Delft, Netherlands (1980).

[22] M. A. Akeroyd and A. Q. Summerfield, "A fully temporal account of the perception of dichotic pitches," Br. J. Audiol., 33, 106-107 (1999).

[23] W. Gaik, "Combined evaluation of interaural time and intensity differences: Psychoacoustic results and computer modeling," J. Acoust. Soc. Am., 94, 98-110 (1993).

[24] T. Djelani, Psychoakustische Untersuchungen und Modellierungsansätze zur Aufbauphase des auditiven Präzedenzeffektes, Dissertation Ruhr-Universität Bochum (2001).

[25] R. Meddis, "Simulation of mechanical to neural transduction in the auditory receptor," J. Acoust. Soc. Am., 79, 702-711 (1986).

[26] R. Meddis, "Simulation of auditory-neural transduction: Further studies,” J. Acoust. Soc. Am., 83, 1056-1063 (1988).

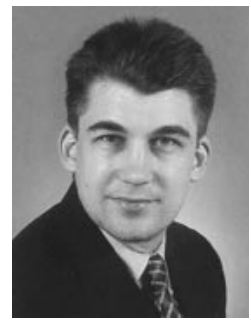

Jonas Braasch was born in 1971 in Wipperfürth, Germany. He received a diploma degree in physics from Dortmund University in 1998 and a Doctor-of-Engineering degree from RuhrUniversity Bochum in 2001. Currently, he is working at the Institute of Communication Acoustics at the Ruhr-University Bochum, managing the group "Auditory Signal Processing and Binaural Technology." His research interests include binaural technology and perception and the acoustics of organs.

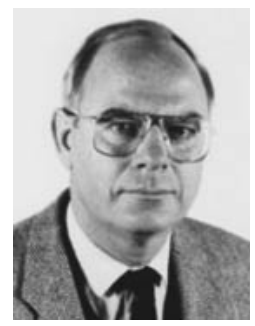

Jens Blauert was born in 1938. He studied communication engineering at Aachen, where he received a Doctor-of-Engineering degree in 1969. In 1973, he delivered an inaugural dissertation to the Technical University of Berlin (habilitation) and in 1994 he was awarded an honorary Dr. technices degree by the University of Aalborg (DK). Since 1974 he holds a chair in electrical engineering and acoustics at the Institute of Communication Acoustics of the RuhrUniversity at Bochum. His major fields of current interest are binaural technology, models of binaural hearing, architectural acoustic, noise engineering, product-sound design, speech technology, virtual environments and telepresence. Currently, he is president of the German Acoustical Society, DEGA. 